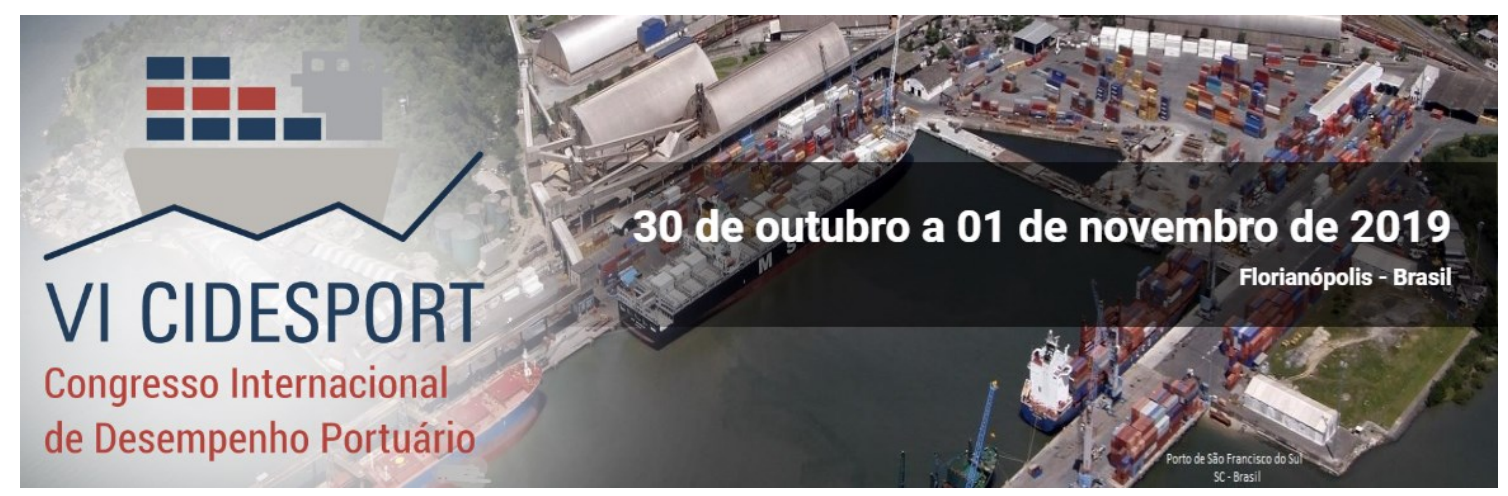

\title{
A CONFIABILIDADE DA ENTREGA DE EQUIPAMENTOS DE PROTEÇÃO INDIVIDUAL - EPI ALIADA A GESTÃO TECNOLÓGICA DE SEGURANÇA DO TRABALHO
}

\author{
Bruno Ambrosi \\ TECON Salvador \\ Fransival Pereira Costa \\ TECON Salvador
}

Resumo: A atualização e otimização dos processos de segurança, demonstra-se oportunidades para todas as empresas, com a inclusão da Ficha eletrônica para controle e gestão dos Equipamentos de proteção Individual - EPI alterados na norma regulamentadora 06 (NR-06) em 2009, abriu possibilidades de melhoria dos processos de segurança referente a este tema. A urgência das empresas por processos mais rápidos e eficientes e a relação do enraizamento de cultura de gestão de Segurança do trabalho tem a busca incessante por inovações tecnológicas e da otimização de tarefas e processos, o que auxiliará para um negócio mais eficiente e com menor custo. O Tecon Salvador baseado em análise de melhoria contínua de seus processos estudou que o controle de Equipamentos de Proteção Individual - EPI utilizado através de uma ficha física sem o respectivo controle correlacionado entre todos os aspectos operacionais de atividade acarretavam em fragilidades de gestão de segurança bem como gasto do recurso. Este trabalho aborda a otimização do processo de gestão de EPI do Tecon Salvador através de um sistema de controle informatizado e entrega por controle biométrico. Com a Informatização do sistema com análise das deficiências em consumo e a projeção de redução de custo na compra de Equipamentos de Proteção Individual - EPI por toda a empresa, contatase que a informatização das ferramentas é investimento de ganho real para a gestão de segurança da empresa e que o primeiro resultado após implementação do sistema, apesar de não conclusivo, geram perspectivas reais de ganhos orçamentais não apenas na gestão de segurança, mas para o resultado da empresa como um todo.

Palavras-chave: Confiabilidade. Gestão. EPI. Redução de custo.

\section{INTRODUÇÃO}

O Tecon Salvador, que iniciou suas operações no ano 2000 , quando foi arrendado pela empresa Wilson Sons. Ocupando uma área arrendada da CODEBA no Porto Organizado de Salvador, o terminal oferece serviços de operação portuária, DEPOT, estufagem e desova, armazenagem alfandegada, tomadas frigoríficas, movimentação e armazenamento de cargas especiais, atuando ainda na carga e descarga de navios de longo curso e de cabotagem, além de armazenamento de cargas de importação e exportação. Além de garantir através de equipe própria de

*A revisão gramatical, ortográfica, ABNT ou APA foi realizada pelos autores. 


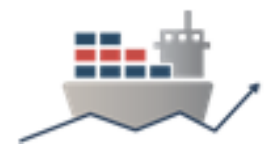

engenhaira e manutenção de equipamento e estruturas físicas. (Portal Tecon Salvador)

O Tecon Salvador é uma das maiores operadoras de serviços portuários, marítimos e logísticos do Brasil, situada em Salvador na Bahia, possui uma área de $118 \mathrm{mil} \mathrm{m}^{2}$, com capacidade para movimentar 530 mil TEU por ano. (Portal Tecon Salvador)

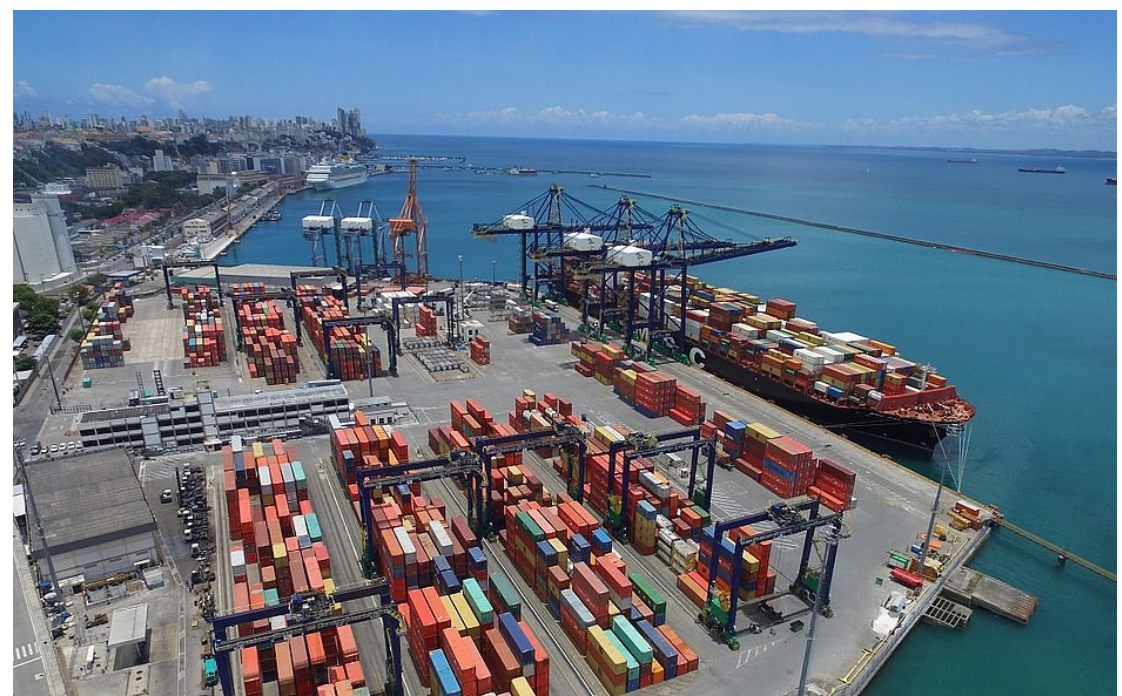

Figura 1: Terminal de contêineres no Porto de Salvador. (Fonte: Tecon Salvador).

O constante crescimento do negócio, implica em maior produtividade, que sugere uma tendência de aumento do risco de acidentes acontecerem. Desta forma a empresa com respectivo alinhamento ao seu planejamento estratégico do Grupo Wilson Sons, onde um dos seus pilares determina "Alcançar e Manter Excelência em SMS" e nesse mesmo contexto também determina a busca pela eficácia, eficiência e inovação nos processo que geram diferenciação para os clientes e/ou ganhos reais, baseado nesta vertente a empresa define que a adequada gestão em Saúde Ocupacional, Meio Ambiente e Segurança do Trabalho (SMS) demonstra sua visão como uma empresa com maior destaque estratégico, sendo que na medida em que reduz o seu risco operacional, melhora o desempenho e prepara melhor para as crescentes demandas da sociedade.

Então, o Tecon atento as mudanças da legislação, aliado a sua busca incessante pela melhoria dos processos, percebeu que informatizar a entrega dos Equipamentos de Proteção Individual - EPI poderia trazer uma melhor gestão na garantia dos processos de SMS para a empresa e para o trabalhador, através da redução de custos e confiabilidade na entrega adequada de Equipamentos de Proteção Individual - EPI a cada funcionários dentro dos requisitos técnicos e legais.

Assim, o objetivo geral deste trabalho foi investigar os resultados alcançados pelo Tecon Salvador com a gestão tecnológica de segurança na entrega dos Equipamentos de Proteção Individual - EPI.

Um estudo desta natureza, justifica-se em virtude dos seguintes objetivos:

- Agilizar a entrega dos Equipamentos de Proteção Individual - EPI;

- Eliminar erros no preenchimento da ficha e na entrega dos Equipamentos de Proteção Individual - EPI;

- Aumentar a confiabilidade das informações de acordo com a matriz de Equipamentos de Proteção Individual - EPI por função e PPRA - Programa de Prevenção de Riscos Ambientais; 
- Garantir que o trabalhador tenha acesso ao Equipamentos de Proteção Individual - EPI adequado aos riscos identificados;

- Gerir os prazos de vida útil dos Equipamentos de Proteção Individual - EPI entregues aos trabalhadores através de sistema seguro e informatizado;

- Diminuir os riscos de processos trabalhistas;

- Reduzir custos;

Para consecução destes objetivos e pelo próprio delineamento do objeto de pesquisa, optou-se por realizar um estudo de caso, com abordagem qualitativa e quantitativa.

\section{CONTEXTO}

A gestão de Equipamentos de Proteção Individual - EPI trata-se de um trabalho voltado para garantir a forma mais adequada, eficaz e tecnológica, para fornecer corretamente, realizar o registro de entrega e incluindo a sua devolução, orientar e treinar sobre seu uso, dentre outras funções especificadas no EPI. Falando sobre Proteção Individual do trabalhador no Brasil, em 1919 surge a primeira lei voltada para acidentes do trabalho, com o Decreto Legislativo $\mathrm{n}^{\circ}$. 3.724, de 15 de janeiro, como ponto de partida da intervenção do Estado nas condições de consumo da força de trabalho industrial em nosso país, onde exige reparação apenas em caso de "moléstia contraída exclusivamente pelo exercício do trabalho, quando este for de natureza a só por si causá-la", instituindo o pagamento de indenização proporcional à gravidade das sequelas. Abre, então, a possibilidade das empresas contratarem o Seguro de Acidente de Trabalho (SAT), junto às seguradoras da iniciativa privada. (CIZS, 2015), no entanto não há registros ou ressalvas sobre uso de Equipamentos de Proteção Individual - EPI nesta legislação citada

A Lei que especificamente tratou assunto Equipamento de Proteção Individual, foi a sétima lei emitida com referência a Segurança do trabalho no Brasil, isso só no ano de 1978 , onde a portaria 3.214 em 08 de julho de 1978 aprova a Lei 6.514 de 22 de dezembro de 1977 que aprova as Normas Regulamentadoras - NR - do Capítulo V, Título II, da Consolidação das Leis do Trabalho, relativas à Segurança e Medicina do Trabalho.

Segundo Cunha (2006) e a Norma Regulamentadora NR-6, Equipamento de Proteção Individual (EPI), refere-se a um equipamento de uso particular, tendo como função a de minimizar certos acidentes e também a proteger contra certas doenças que poderiam ser ocasionadas pelo ambiente de trabalho.

O Tecon Salvador possui em torno de 620 trabalhadores, com pelo menos 120 funções diferentes o que gera uma alta demanda para a determinação de riscos de acordo com a gama de atividades específicas para cada descrição de atividade.

Dentro desse dessa sequência de determinação do risco, fornecimento do Equipamentos de Proteção Individual - EPI, registro de entrega e devolução, foi percebida falhas de controle que deixavam vulnerabilidades no sistema, além de onerar o custo por falha no processo de gestão.

Em 2009, através da portaria SIT (Secretaria de Inspeção do Trabalho/Departamento de Segurança e saúde no trabalho) número 107, alterou a alínea 6.61 da Norma NR:06 para o seguinte texto: "h) registrar o seu fornecimento ao trabalhador, podendo ser adotados livros, fichas ou sistema eletrônico.", possibilitando que o controle de entrega fosse através de meio eletrônico. 


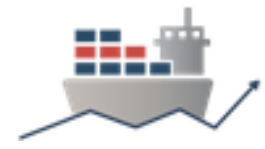

Através de análise feita em amostragem de fichas de Equipamentos de Proteção Individual - EPI do Tecon Salvador foi constatada a deficiência existente no processo nos seguintes itens:

- Discrepâncias nas informações transcritas nas fichas de Equipamentos de Proteção Individual - EPI;

- Tempo muito longo/curto para substituição dos Equipamentos de Proteção Individual - EPI;

- Ausência do preenchimento do Certificado de Aprovação dos Equipamentos de Proteção Individual - EPI disponibilizados transcritos na ficha;

- Não preenchimento da devolução dos Equipamentos de Proteção Individual EPI;

- Rasuras na documentação, dificultando legitimidade ao documento;

- Falta de Assinatura do colaborador na ficha de Equipamentos de Proteção Individual - EPI;

- Retirada repetidas do mesmo Equipamentos de Proteção Individual - EPI sem critério de prazo;

Para melhorar toda a gestão da entrega e controle dos Equipamentos de Proteção Individual - EPI consumidos no Tecon, foi submetido para implementação o programa de gestão através de biometria de entrega de Equipamentos de Proteção Individual - EPI que ao qual foi concluída sua implementação plenamente no mês de julho/2019.

\section{INTERVENÇÃO}

O Tecon Salvador continuamente busca a inserção de seus processos a sistemas de automatização e de atendimento ao cliente de forma digital. Alguns exemplos de tecnologias aplicadas ao terminal são:

- Portal do cliente;

- Acesso de caminhões com biometria;

- Reconhecimento ótico de caracteres em containners;

- Sistema de gestão integrado Navis N4;

Considerando como não mais uma tendência e sim uma realidade, a gestão tecnológica foi disseminada para evolução constante em todos os setores da empresa, desta forma foi necessário perceber onde haviam possibilidades, dentro da gestão de segurança do trabalho, de ganhos tecnológicos, mais eficazes para atendimento a requisitos legais, mas principalmente os ganhos com redução de custos atrelado ao consumo de Equipamentos de Proteção Individual - EPI.

Após o levantamento das necessidades e análise foi percebido a possibilidade de informatização da gestão de controle de Equipamentos de Proteção Individual $\mathrm{EPI}$, com controle parametrizado através de biometria, sistema já existente e implementado para gestão de acesso de caminhões.

Aparentemente um projeto simples porém a falhas nos controles foi percebida devido a inconsistências durante levantamento de evidências para perícias judiciais, ao qual os itens só eram identificados em caso como essas solicitações periciais.

Com o viés de melhoria da gestão o Tecon Salvador, implementou o projeto de gestão de Equipamentos de Proteção Individual - EPI com o objetivo instalação de aparelhos com leitura biométrica com interação ao sistema já existente no Tecon Salvador "NEXO CS", no qual é capaz de realizar o registro individual de cada 
funcionário no momento em que o Equipamentos de Proteção Individual - EPI for retirado e/ou devolvido, fazendo um registro mais eficaz, confiável e ágil. Após análise do custos de consumo e respectivas deficiências no sistema de gestão de EPI levantados e analisados, foi aplicado um pequeno investimento (em torno de $\mathrm{R} \$ 3.000,00$ ) para parametrização do sistema atual de controle de estoque ao controle de entrega de Equipamentos de Proteção Individual - EPI.

Com a gestão sistematizada e individual, permite-se que seja possível analisar o quantitativo de tempo de durabilidade de um Equipamentos de Proteção Individual - EPI, bem como entrega dos Equipamentos de Proteção Individual - EPI apenas ao qual estão direcionados ao risco, garantindo uma gestão adequada.

O projeto foi desenvolvido em 6 principais fases:

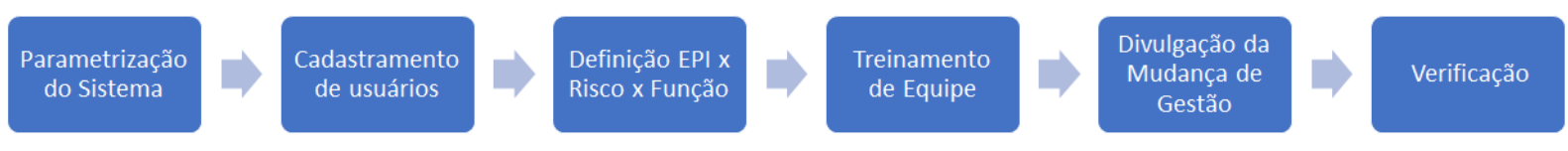

Figura 2: Fluxo de implementação do Projeto .

A parametrização foi a primeira etapa do projeto que consistiu na interação entre os sistema de Estoque, realizado através do SAP/ERP (SAP/ERP o sistema integrado de gestão empresarial com módulo específico de controle de estoque), junto ao NEXO CS (Sistema de gestão de segurança e saúde ocupacional) ao qual já havia um módulo disponível de biometria para gestão de Equipamentos de Proteção Individual - EPI. O período de implementação desta etapa, foi em torno de 1 mês, salienta-se que este tempo curto está atrelado a empresa já possuir sistemas que eram compatíveis entre si, facilitando a agilidade na implementação.

A parametrização resumiu-se em 3 Principais pontos:

- Testes de Compatibilização entre sistemas;

- Aquisição de leitores biométricos;

- Instalação do leitores biométricos;

- Treinamento dos Operadores do sistema;

Após parametrização confirmada, foi sequenciada a etapa de cadastramento. A etapa apesar de simples, foi exigida um planejamento conjunto entre as equipes de cadastros e as áreas demandadas, esta é a etapa que gerou uma maior demanda de tempo devido a empresa possuir em torno de 620 funcionários em trabalho dividido em 3 turnos, para implementação desta etapa as ações foram divididas da seguinte forma:

- Planejamento para Cadastro

- Cadastro biométrico

- Assinatura de Termo de Conhecimento e Autorização

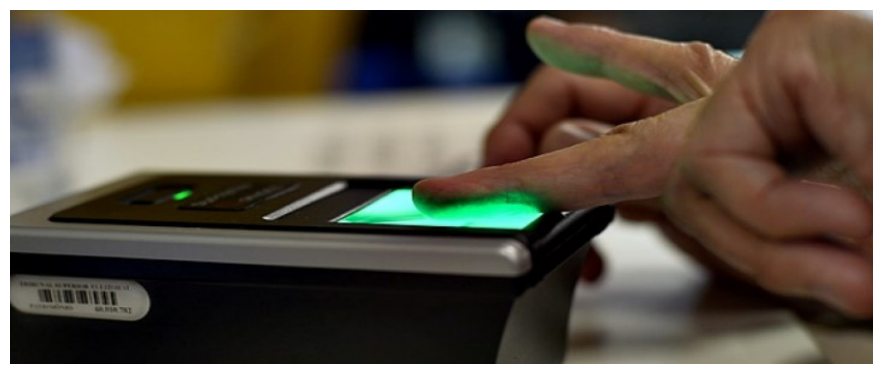




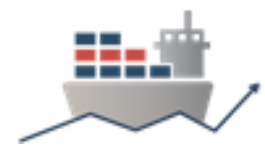

Figura 3: Cadastramento Biométrico.

A principal preocupação referente a implementação seria o passivo referente a autorização e ciência pelo demandante referente formalização da biometria como uma assinatura com validade jurídica, desta forma foi realizada a inclusão de um termo de conhecimento e autorização, elaborado especificamente para dar conhecimento ao cadastrado sobre a gestão biométrica, bem como garantir a autorização do mesmo com relação a "assinatura digital biométrica".

A etapa subsequente foi de determinar quais Equipamentos de Proteção Individual - EPI deveriam estar parametrizados conforme função e riscos ao qual cada funcionários estão expostos, período para implementação desta etapa foi considerada rápida na elaboração das matrizes de $\mathrm{EPI}$, no entanto, visando uma o comprometimento e avaliação do responsável da área foi necessário garantir sua validação pelos mesmos.

Otimizando o controle de gestão de Equipamentos de Proteção Individual EPI. Para essa etapa o principal desafio foi a de levantamento dos Equipamentos de Proteção Individual - EPI por áreas: Operações, Manutenção, Engenharia, Administrativo/ Financeiro, Tecnologia da Informação e qualidade de processos (TIQP), Desenvolvimento Humano e organizacional (DHO), Comercial, Segurança, Meio ambiente, Saúde e patrimonial (SMSP). A etapas de implantação desta etapa se dividiram da seguinte forma:

- Elaboração de Matriz de Equipamentos de Proteção Individual - EPI por Função;

- Validação da matriz por Gestores;

- Parametrização de Equipamentos de Proteção Individual - EPI por função no sistema;

As etapas de treinamento e divulgação, que levou em torno de 1 mês, estão relacionadas com a direta perenização da mudança em todos os segmentos da empresa, através de treinamento dos operadores do sistema e garantindo o conhecimento do novo processo de gestão biométrica para toda força de trabalho.

A última etapa, que se trata de uma etapa contínua e periódica, consistiu em revisão das Auditorias de Equipamentos de Proteção Individual - EPI, com adequação a verificação ao novo sistema:

- Revisão do formulário (Quesitos específicos - Biometria);

- Verificação de conformidade do sistema;

- Auditoria de Inspeção;

Em resumo do projeto consistiu na implantação de aparelhos com leitura biométrica, através de programa já em uso no Tecon Salvador "NEXO CS", no qual seja capaz de realizar o registro individual de cada funcionário no momento em que o Equipamentos de Proteção Individual - EPI for retirado e/ou devolvido, fazendo um registro mais eficaz, confiável e ágil. No entanto dando condições de acompanhamentos mensais de controle e gestão do consumo e entrega dos EPI's.

\section{RESULTADOS OBTIDOS}

A implementação do Projeto de Biometria de Equipamentos de Proteção Individual - EPI trouxeram como benefícios imediatos para o Tecon Salvador:

- Eliminação de fichas físicas ou controles paralelos em excel;

- Agilidade e rapidez no processo de entrega; 
- Gestão eficaz dos registros de retirada e devolução de Equipamentos de Proteção Individual - EPI;

- Agilidade na busca de evidências para perícias trabalhistas;

- Redução de custo significativa pelo consumo de papel;

- Confiabilidade das informações;

- Levantamento de ofensores por consumo de Equipamentos de Proteção Individual - EPI;

- Principais Equipamentos de Proteção Individual - EPI utilizados por período;

- Controle de validade do Equipamentos de Proteção Individual - EPI e do Certificado de Aprovação (C.A.).

Sob a ótica de custos, ainda não há parâmetros para uma constatação de ganhos efetivos e imediatos, porém o mês de agosto com 2019 demonstrou-se com uma redução de $22 \%$ (Conforme Tabela 1) em comparação com o mesmo mês do ano anterior. O que se demonstra que houve uma redução significativa dos custos de materiais de segurança no mês de plena operação do sistema. Convém que haja melhor apuração dos dados de custos para uma melhor apuração da eficácia em redução com a implementação do sistema, em se tratando de um Terminal portuário, há sazonalidades de atividades que podem gerar o consumo de EPI's específicos para atividades esporádicas que podem gerar custos adicionais não mapeados.

Tabela 1 - Consumo de Materiais de segurança em 2018- 2019 (Fonte: Tecon Salvador).

\section{8}

\begin{tabular}{c|cccccccccccc}
\hline & Jan & Fev & Mar & Abr & Mai & Jun & Jul & Ago & Set & Out & Nov & Dez \\
\hline $\begin{array}{c}\text { Consumo } \\
E P I \\
(R \$)^{*}\end{array}$ & $\mathbf{2 1 . 7 k}$ & $19.1 \mathbf{k}$ & $\mathbf{2 2 . 9 k}$ & $\mathbf{3 5 . 2 k}$ & $\mathbf{1 6 . 6 k}$ & $\mathbf{1 9 . 5 k}$ & $\mathbf{2 1 . 2 k}$ & $\mathbf{2 5 . 6 k}$ & $\mathbf{2 4 . 0 k}$ & $\mathbf{2 7 . 8 k}$ & $\mathbf{1 9 . 4 k}$ & $\mathbf{1 7 . 0 k}$ \\
& & & & & & & & & & & & \\
\hline
\end{tabular}

2019

\begin{tabular}{c|cccccccccccccc}
\hline & Jan & Fev & Mar & Abr & Mai & Jun & Jul & Ago & Set & Out & Nov & Dez \\
\hline $\begin{array}{c}\text { Consumo } \\
\text { EPI } \\
(\mathrm{RS})^{*}\end{array}$ & $\mathbf{2 9 . 5 k}$ & $\mathbf{2 9 . 5 k}$ & $\mathbf{2 4 . 5 k}$ & $\mathbf{2 4 . 1 k}$ & $\mathbf{5 6 . 2 k}$ & $\mathbf{2 5 . 7 k}$ & $\mathbf{3 1 . 6 k}$ & $\mathbf{1 9 . 8 k}$ & - & - & - & - \\
\end{tabular}

De um modo geral, a gestão informatizada do sistema de gestão de Equipamentos de Proteção Individual - EPI. Por outro lado, percebeu-se que o trabalhador vem tendo mais informação, conscientização sobre segurança e a ergonomia do equipamento de proteção, o que garante o melhor uso dos equipamentos.

\section{CONSIDERAÇÕES FINAIS}

Com base no estudo realizado pelo Tecon Salvador, onde se priorizou questões sobre a gestão de Equipamentos de Proteção Individual - EPI através determinação do risco, fornecimento do Equipamentos de Proteção Individual - EPI, registro de entrega e devolução, foi percebida falhas de controle que deixavam 
vulnerabilidades no sistema, além de onerar o custo por falha no processo de gestão, constatou-se que houve ganhos imediatos com a implantação, através da confiabilidade das informações, agilidade e redução de impressão e controles de validades. Importante destacar que através da informatização do controle trona-se disponíveis informações que podem ser objeto de trabalhos pontuais para redução de custo como o controle de EPI por função e tempo de troca, quantificar o consumo por área ou por Equipamentos de Proteção Individual - EPI consumido.

Com isso considera-se que a gestão de biometria de entrega de Equipamentos de Proteção Individual - EPI, se faz eficiente, e que também é possível realizar uma gestão de orçamento mais eficaz, o nível percentual de redução alcançável para os custos ainda não são mensuráveis, no entanto, o resultado do primeiro mês de implementação plena do sistema demonstra-se promissor.

\section{REFERÊNCIAS}

BRASIL. Ministério do Trabalho. NR 6 - Equipamento De Proteção Individual - EPI. Disponível em: https://enit.trabalho.gov.br/portal/images/Arquivos_SST/SST_NR/NR06.pdf Acesso em 31/08/2019.

BAHIA DE VALOR. Portos públicos da BA registram o melhor resultado da história. 18/01/2018

Disponível em: https://www.bahiadevalor.com.br/2018/01/portos-publicos-daba-registram-o-melhor-resultado-da-historia Acessado em: 31/08/2019.

CISZ, CLEITON RODRIGO . Conscientização Do Uso De Epi's, Quanto À Segurança Pessoal E Coletiva. 2015. 44 p. Monografia (Especialização em Engenharia de Segurança do Trabalho)- Universidade Tecnológica Federal do Paraná, CURITIBA, 2015.2 Disponível em: http://repositorio.roca.utfpr.edu.br/jspui/bitstream/1/3833/1/CT_CEEST_XXIX_2015_ 07.pdf . Acesso em: 31/08/2019.

CUNHA, Marco Aurélio Pereira da. Análise do uso de EPI's e EPC's em obras verticais. Tese (Especialização em Engenharia de Segurança do Trabalho) Universidade Federal de Mato Grosso, Cuiabá, 2006.

NETO, Nestor Waldhelm. O que é EPI - Equipamento de Proteção Individual. Disponível em : http://segurancadotrabalhonwn.com/o-que-e-epi/.

Portal Tecon Salvador. Logística 4.0: um salto de produtividade. Disponível em: https://www.wilsonsons.com.br/pt/teconsalvador/noticia/logistica-40-um-salto-deprodutividade. Acesso em: 26 de agosto de 2019.

Portal Tecon Salvador. Quem Somos. Disponível em:

https://www.wilsonsons.com.br/pt/teconsalvador/conteudo/quem-somosts. Acesso em: 30 de agosto de 2019

WIKIPEDIA.ORG. Indústria 4.0. Disponível em:

https://pt.wikipedia.org/wiki/lnd\%C3\%BAstria_4.0 Acesso em: 23 de agosto de 2019. 
VI CIDESPORT/2019

Congresso Internacional

de Desempenho Portuário

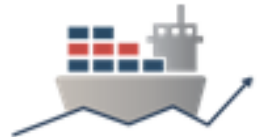

Revista CIPA. A inovação no sistema de gerenciamento de EPIs: 3M Connected Safety. Disponivel em: http://revistacipa.com.br/inovacao-no-sistema-degerenciamento-de-epis-3m-connected-safetyl Acesso em: 02 de setembro de 2019. 